(1)

George Fox

UNIVERSITY
Digital Commons @ George Fox University

Faculty Publications - School of Physical

Therapy

School of Physical Therapy

6-1987

Using Neuromuscular Electrical Stimulation to Facilitate Limb

Control in the Head-Injured Patient

Cynthia Zablotny

Follow this and additional works at: https://digitalcommons.georgefox.edu/pt_fac

Part of the Physical Therapy Commons 


\section{Using neuromuscular electrical stimulation to facilitate limb control in the head-injured patient}

Cynthia Zablotny, BS, PT

Physical Therapy Clinical Instructor Adult Head Trauma Service

Rancho Los Amigos Medical Center Downey, California
NEUROMUSCULAR electrical stimulation 1 (NMES) has been used to treat a variety of extremity problems in head-injured patients. Specifically, NMES has been used to gain motor control, reduce joint contractures, and reduce muscle hypertonicity. ${ }^{1}$ Such uses rely on intact peripheral nerve excitability to generate the desired muscular response.

This article will present some clinically relevant methods of maximizing the effectiveness of NMES programs for facilitating limb control in the head-injured patient. Stimulator features that help ensure treatment success will be identified. The special cognitive considerations that must be addressed when using NMES on the head-injured patient will also be discussed. The article will conclude with a discussion of therapeutic and functional tasks that NMES may help facilitate.

\section{STIMULATOR PARAMETERS AND FEATURES}

In order to increase the ease of acceptability of clinical NMES programs for the headinjured patient, the therapist needs to choose stimulation units that exhibit desirable param- 
eters and features. Since lengthy explanations of the stimulation program are not possible with most head-injured patients, much of the patient's reaction to this modality will rely on his or her perceived comfort of the stimulation. Variables that should be considered when designing NMES programs for the headinjured patient are discussed below.

\section{Current waveform}

The majority of commercially available units deliver a pulsatile current featuring one of two waveform configurations. A compensated monophasic waveform (also referred to as an asymmetrical biphasic waveform) is useful when a discrete response is required in a relatively small muscle, as may be the case in many NMES programs for the upper extremity and the ankle joint. ${ }^{2}$ Excitation of the desired neuromuscular response occurs primarily under the negative electrode with this type of current waveform. ${ }^{2}$ The advantage of this waveform is that once the motor point of the small muscle is located and the active electrode placed over it, the current amplitude can be kept at a minimum while a discrete muscle contraction is generated. This is especially important for the head-injured patient since it limits some of the unpleasant sensory experiences associated with surface stimulation.

Units that produce a symmetrical biphasic waveform are indicated for large muscle activation. This type of waveform causes excitation under both electrodes, enabling the recruitment of more muscle fibers in the response. ${ }^{2,3}$ Two separate motor points must be designated for electrode placement to ensure that the best possible stimulated contraction is achieved. Because more muscle fibers can be recruited with this waveform, less current amplitude is needed to generate the same quality of large muscle contraction than would be required if a compensated monophasic waveform was used. ${ }^{3}$ This feature makes the symmetrical biphasic waveform an efficient one for stimulating muscles such as the quadriceps femoris, gluteus maximus, or gluteus medius. It also lessens the probability that sensory intolerance will develop. Normal subjects have shown a preference for this waveform over the compensated monophasic type when stimulation was delivered to a large muscle group. ${ }^{3}$

\section{Ramp up time}

The patient's comfort with the stimulation can be enhanced by employing a ramp up (rise) time to the train of current pulses. This stimulation parameter adjusts the time allowed for the pulse train to reach its set amplitude, thus controlling the abruptness of the stimulated response. A minimal ramp up time of 2 seconds is recommended, although certain patients may require more time to ensure their comfort. ${ }^{2}$ The ramp up time may need to be prolonged initially during the early training stages of the NMES program so that the patient may accommodate to the sensations associated with surface stimulation. A prolonged ramp up time of at least 6 to 8 seconds may also be indicated if the stimulated muscle group opposes a spastic antagonist, ${ }^{2}$ which will help avoid the imposition of a quick stretch to the opposing musculature. If available, a ramp down or fall time can also improve the overall comfort of the stimulated muscle contraction by modulating the time it takes for this response to end.

\section{External triggers}

Since many of the NMES programs used with head-injured patients involve facilitating a specific and timely response, a unit with external triggers is desirable. ${ }^{1}$ Hand or foot switches add immensely to the versatility of a neuromuscular electrical stimulator, especially when designing treatment programs for head-injured patients. Stimulation 
units that provide only cyclical stimulation can be useful in strengthening or contracture reduction programs. However, in a facilitation program, the therapist needs to be able to trigger the unit at a precise time so that the stimulated input is presented in a logical and meaningful manner. It may be impractical to have the head-injured patient try to match his or her movement with a cycling stimulator, especially if the patient is performing some type of functional task. Variations in the patient's response, whether it be cognitive or sensorimotor in nature, will not be accommodated. As a result, the input from the stimulator may be perceived as confusing rather than helpful. Facilitation responses during gait necessitate the use of an external trigger so that the sequence and timing of the stimulation coincides with the appropriate point in the gait cycle.

\section{COGNITIVE CONSIDERATIONS}

An important consideration before initiating a stimulation program with a head-injured patient is his or her cognitive status. The patient's level of cognition will greatly influence the appropriateness of using this modality to achieve some therapeutic effect. Patients who are extremely confused and agitated (cognitive level IV) are not NMES candidates since the sensory input from the stimulation may further add to their confusion in processing information, ${ }^{1,4}$ Likewise, patients demonstrating a localized response to an external stimulus (cognitive level III) are not candidates for an NMES program since they may have a heightened response to the stimulation and perceive it as noxious. ${ }^{1,4}$

Some of the more traditional programs using NMES for the facilitation of motor control require active participation on the part of the patient. This type of program may not be effective for patients with limited attention spans or those who are easily distracted. How- ever, as cognition improves and the patient demonstrates an improved ability to attend to a task, this type of program may serve to facilitate motor control and sharpen the patient's attention skills concomitantly.

Certain NMES programs do not require the patient to be attentive to the stimulation throughout its duration. This is true if the patient is receiving cyclical stimulation for contracture reduction or muscle strengthening. In this case, cyclical electrical stimulation can be used while the patient attends other activities throughout the day. An adequate NMES training program would ensure that the patient could tolerate the stimulation without it interfering with his or her attentiveness in other therapies.

The training period is designed to gradually acclimate the patient to the sensations associated with surface stimulation. During this time, the amplitude of the stimulation is gradually increased to the patient's tolerance until the desired contraction level is achieved. ${ }^{2}$ It may take approximately one week to reach this goal in some patients. An additional benefit of this type of gradual training is that the stimulated muscles have time to build up some resistance to fatigue. Such resistance is especially important if the patient is to be put on an NMES program involving multiple hours of stimulation per day.

\section{FACILITATION PROGRAMS USING NMES}

Facilitation programs using NMES can easily be combined with more traditional therapies. The purpose of combining these therapies is to accomplish one or more of the following goals:

- to improve the patient's awareness of his or her extremity or its desired movement;

- to improve the timing of the muscular response so that the movements may be smooth and coordinated; and 
- to augment the quality of the patient's own muscular response so that the functional or therapeutic demands of a task can be completed.

The use of low amplitude stimulation sufficient to provide a sensory cue can be especially helpful when working with head-injured patients exhibiting extremity sensory losses, absent or delayed initiation of a motor response, or neglect of a body part. In order for this type of stimulation program to be successful, the patient must have adequate motor control to complete a given task once the submotor sensory cue is provided.

Patients lacking adequate volitional control to complete a given functional or therapeutic task can benefit from extremity stimulation provided at amplitudes sufficient to create a motor response. The effectiveness of surface stimulation programs in augmenting the strength of the patient's volitional response depends on the availability of the target muscle for electrode placement and the sensory tolerance of the patient. Even with extremely thin patients, it is difficult to generate a fair contraction of a muscle such as the gluteus maximus using surface electrodes. ${ }^{2}$ Overlying adipose tissue limits the stimulated response of this muscle group. The gluteus medius presents a different problem in that a relatively small part of the muscle is superficial enough for surface electrode activation. ${ }^{2}$ Despite these limitations, the stimulated contractions of such muscle groups may be enough to at least supplement the patient's volitional efforts. Other muscles, such as the hip flexors, lie too deep for surface electrode activation.

Head-injured patients frequently demonstrate difficulty in coordinating their limb movements so that a fluid response is attained. Specific responses may be delayed because of sensory and/or motor deficits. NMES programs providing sensory input only or combining both a motor and sensory response can help the patient properly coordi- nate his or her movements to complete a therapeutic or functional task. For example, triggered stimulation may enhance the patient's ability to demonstrate a smooth reciprocation of movement as upper or lower extremity diagonal proprioceptive neuromuscular facilitation (PNF) patterns are performed.

\section{Encouraging lower extremity weight bearing}

Stimulation of key lower extremity muscle groups can enhance the patient's ability to maintain stability and limb alignment as progressive weight-bearing activities are attempted. The patient may initially begin to accept weight on his or her lower extremities with the use of a tilt table. Quadriceps, hip extensor, or abductor stimulation may be used initially to cue the patient to tighten these muscles when upright. As trunk and limb control improves, the patient may progress from tilt table activities to standing with either the support of the therapist or an external device such as a standing frame. NMES to these same muscles is easily incorporated into this activity. The use of an external hand switch triggered by the therapist is helpful in this situation because the therapist can appropriately time the onset of the stimulation with the patient's response to verbal or tactile cues to shift weight onto the stimulated limb. The therapist may find this type of NMES program beneficial as the patient begins ambulation since it may ready the patient to superimpose limb advancement skills over this basic stance stability.

\section{Gait training}

Patients with residual problems in hip extensor control, coupled with inadequate tibial control, are often excellent candidates for combining orthotic management strategies with NMES. Typically, such patients collapse into inadequate hip and knee extension during the single limb support phase of gait. This results in a markedly increased energy expen- 
diture with ambulation. Despite the use of an ankle foot orthosis, inadequate limb extension often persists. NMES to the hip extensors or quadriceps can eliminate this flexed posture, thus reducing the energy cost of the task.

Head-injured patients often exhibit problems in hip abductor control that results in a contralateral pelvic drop. This gait deviation can be especially devastating for the headinjured patient: His or her dynamic balance responses may be challenged beyond their abilities as the pelvis rapidly drops in single limb support. Hip abductor stimulation, trig. gered to commence before the patient demonstrates the dropped pelvic posture, can help maintain pelvic alignment and stance stability during single limb support. Because it would be ineffective if triggered after the pelvis has already assumed its dropped position, the stimulated contraction must be carefully timed.

NMES may also be incorporated into gait training programs designed to enhance control of the swing phase. As previously stated, successful hip flexor surface stimulation is not possible because of the location of these muscles. Hamstring surface stimulation is also not successful in improving knee flexion in swing since it is not possible to isolate a contraction of the short head of the biceps femoris without concomitantly activating the long hamstrings. As a result, unwanted hip extension is also generated. However, in certain patients, it is possible to achieve some limb advancement capabilities through activation of the peroneal nerve to generate a total limb flexion response. If inadequate dorsiflexion control is the primary swing phase problem, stimulation of these muscles can be used to achieve a neutral ankle position to help in limb clearance in midswing and to properly position the ankle so that a heel contact can be made. The use of a foot switch placed in the forefoot of the patient's shoe can be an effective means of timing the stimulation. Each patient must be carefully evaluated by the therapist to see how reliable the foot switch is in providing consistent stimulation at the correct point in the gait cycle.

It is often difficult for the head-injured patient to achieve complete knee extension in terminal swing because of hamstring spasticity or synergistic movement. Knee extension stimulation triggered by the therapist to occur in terminal swing can help the patient achieve adequate step length and position the limb properly for weight acceptance. This type of stimulation program can help facilitate selective control in the limb since it encourages knee extension to occur comcomitantly with hip flexion.

\section{Mat activities}

Upper and lower extremity NMES programs can be combined with a variety of mat activities to facilitate the desired response. NMES can be used when functional activities such as rolling are performed. Stimulation may remind the patient to include the involved upper or lower extremity in the rolling effort. Elbow extensor stimulation may help the patient maintain correct limb positioning as he or she attempts a transitional movement such as moving from supine to sitting while bearing weight through an extended arm. Triceps stimulation may also be used to help the patient maintain a stable arm posture while crawling activities are attempted. If the coordination of limb movement is the primary problem interfering with forward crawling, submotor stimulation to the shoulder flexors can remind the patient to move the involved arm forward to continue the crawling sequence. Stimulation of the hip extensor or abductor muscles may enhance the patient's stability in assuming a kneeling or half kneeling position.

NMES can be a versatile addition to other therapeutic efforts to improve limb function in the head-injured patient. The effectiveness of 
the combined treatment efforts relies on the therapist's knowledge of and comfort with
NMES and its application in a variety of treatment situations.

\section{REFERENCES}

1. Baker LL, Parker K, Sanderson D: Neuromuscular electrical stimulation for the head-injured patient. Phys Ther 1983;63:1967-1974.

2. Benton L, Baker LL, Bowman BR, et al: Functional Electrical Stimulation-A Practical Clinical Guide, ed 2. Downey, Calif, Professional Staff Association of Rancho Los Amigos Hospital, Inc, 1981.

3. Bowman BR, Baker LL: Effects of waveform parameters on comfort during transcutaneous neuromuscular electrical stimulation. Ann Biomed Eng 1985;13:59-74.

4. Hagen C, Malkmus D, Durham $P$, et al: Levels of cognitive functioning, in Rebabilitation of the Head Injured Adult: Comprebensive Pbysical Management. Downey, Calif, Professional Staff Association of Rancho Los Amigos Hospital, 1979, pp 87-89. 